\title{
Diaspora and Health? Traditional Medicine and Culture in a Mexican Migrant Community
}

\author{
Anna Waldstein*
}

\begin{abstract}
As members of the Mexican diaspora acculturate/assimilate to life in the United States they gain skills that help them improve their socioeconomic status and overcome barriers to the mainstream American healthcare system. Thus, we might expect better health among more acculturated Mexicans. However, most of the research conducted during the past 20 years shows that the health of Mexicans living in the United States deteriorates as acculturation increases. This suggests that certain health promoting aspects of Mexican culture are lost as migrants adapt to and adopt American ways of life. This paper is the first step in testing the hypothesis that declining health among acculturated people of Mexican descent is related to a loss of traditional medical knowledge.
\end{abstract}

During an ethnographic study of women's medical knowledge in an unacculturated Mexican migrant community in Athens, Georgia, I observed many ways low-income, undocumented migrants maintain good health. Migrant women encourage health-promoting behaviors and treat sick family members with a variety of home remedies that appear to be effective according to chemical and pharmacological analyses. Additionally, migrant women in Athens learn how to navigate the American medical and social service systems and overcome barriers to professional healthcare services using information provided through social networks. Nevertheless, migrant women often prefer to treat sick family at home and indicated a preference for Mexican folk medicines over professional medical care in most situations.

This case study suggests that migration and diaspora need not always lead to disease. The maintenance of a Mexican culture that is distinct from the rest of American society helps ensure that traditional medical knowledge is

* Department of Anthropology, University of Kent.

Published by Blackwell Publishing Ltd., 9600 Garsington Road, Oxford OX4 2DQ, UK, and 350 Main Street, Malden, MA 02148, USA. (c) 2008 The Author Journal Compilation (c) 2008 IOM International Migration Vol. 46 (5) 2008

ISSN 0020-7985 
not lost, while the social networks that link Mexicans to each other and to their homeland help minimize threats to health, which are usually associated with migration. Thus, increased access to professional medical care may not improve the health of migrants if it comes with the loss of traditional medical knowledge.

\section{INTRODUCTION}

While migration has become an integral part of modern globalization, it is as old as human society. Unfortunately, morbidity and mortality are associated with both voluntary and forced migration. Most migrants have had to adapt to poor ecological and economic conditions at home and abroad that put them at increased risk of disease and death. In many parts of the world migrants become isolated and suffer infectious and chronic illnesses, work-related injuries and depression (Carballo and Nerukar, 2001). Migrants get sick for many reasons; they receive little medical care and often find themselves living and working in poor (i.e. overcrowded, unsanitary, toxic) environments that are often associated with malnutrition. Moreover, discrimination against migrants in host societies contributes to unhealthy lifestyles and the migration experience itself is inevitably a source of great stress (Bruhn, 1997). Female migrants may face extra challenges related to reproductive health, cultural practices and attitudes toward women (Carballo and Nerukar, 2001).

When diasporas emerge from long-term migration flows, the impacts on human health may become even more pronounced. The definition of diaspora has evolved greatly since it was first used to describe the Babylonian exile of the Jews. However, most scholars agree that contemporary diasporas share three fundamental elements: dispersion of a population in space, homeland orientation, and the preservation of an identity and culture that is distinct from that of the host society (Brubaker, 2005). This latter characteristic contributes to the marginalization and social exclusion of diaspora communities, which can in turn breed poverty and threaten health.

Although the Mexican diaspora has been described as incipient, discrimination against immigrants and their descendents, the close proximity to Mexico, and the development of family and migration networks have maintained a distinctly Mexican culture within the United States (Gonzalez Gutierrez, 1999). Members of the Mexican diaspora face a variety of factors that put them at increased risk of disease. Most Mexicans in 
the United States are poor, which makes it likely that they live in communities with high unemployment and poor housing, schools, social welfare and healthcare facilities (Molina et al., 1994). They are also one of the most educationally disadvantaged groups in the United States and are more likely to work in low-skilled, lower-paying positions (Iannota, 2003). Immigrants who do find work in the United States are overrepresented in hazardous occupations and industries, such as factory and agricultural work, and construction, which put them at greater risk for occupational health problems (Friedman-Jimenez and Ortiz, 1994; Morales et al., 2002).

Migrating to the United States without a visa or work permit further increases threats to health because migrants who do so have few legal rights to social welfare and healthcare services. Undocumented migrants from Mexico are generally viewed as lawbreakers who occupy jobs that belong to American citizens and make excessive use of public assistance programs. This stereotype has lead to government and public support for reducing migration through unilateral measures such as border vigilance and the construction of walls, curtains and fences. These measures restrict all migrants' access to health care, which can contribute to the spread of disease (Bronfman, 1998). Moreover, extreme measures of migration control can also have adverse effects on Mexican Americans because they encourage xenophobia, prejudice and discrimination toward all people of Mexican descent, regardless of their nationality (Gutierrez, 1999).

As Mexican immigrants and their descendents acculturate and assimilate to life in the United States they gain skills that should help them overcome many of the health risks that they face. Acculturation is a correlate of socioeconomic position and Hispanic immigrants who are more acculturated have higher levels of education and income, and are more likely to use primary health care services (Molina et al., 1994). Thus, we might expect better health among more acculturated people of Mexican descent. However, most of the research conducted during the past 20 years shows that the health status of Mexicans living in the United States deteriorates as acculturation increases.

For example, there is evidence that women who are more "Mexican oriented" (i.e. speak Spanish, identify themselves as Mexican and were born in Mexico or had a parent born in Mexico) are less likely to have low birth weight babies than women of Mexican descent who adhere to American cultural norms (Scribner and Dwyer, 1989). According to data 
from the third National Health and Nutrition Examination Survey (NHANES III) Mexican immigrants have the best cardiac profile (Sundquist and Winkleby, 1999) and the lowest prevalence of gallbladder disease (Tseng et al., 2000) compared to Mexicans born and raised in the United States. Likewise, rates of cancer, high blood pressure and teen pregnancy all increase with acculturation (Vega and Amaro, 1994).

Social scientists have attempted to explain this phenomenon by examining the adoption of new behaviours that contribute to increased morbidity and mortality such as drinking, drug use and poor dietary habits. For example, it has been shown that acculturated Mexican women smoke more than women who have been in the United States for shorter periods of time (Marin et al., 1989). There is also evidence that Mexican American men and women consume more alcohol (Morales et al., 2002), are more likely to use illicit drugs (Amaro et al., 1990) and eat poorer diets (Guendelman and Abrams, 1995) than their less acculturated immigrant counterparts.

Missing from the literature on acculturation and health among people of Mexican descent is a consideration of behaviours that may be lost. The World Health Organization has recognized the importance of traditional medical knowledge held by people across the globe as a viable resource for protecting health and meeting primary health care needs. Moreover, the efficacy of such traditional medical beliefs and practices is confirmed by the vast literature in the fields of ethnomedicine, medical ethno-botany and ethno-pharmacology (see Waldstein and Adams, 2006 for a recent review). However, this knowledge may be lost as people migrate to industrialized countries (Pieroni et al., 2005). Thus, a reasonable hypothesis about the association between increased acculturation and declining health status among people of Mexican descent would be that as American culture is adopted Mexican medical knowledge, beliefs and practices that have traditionally protected health are abandoned.

This paper is a first step in testing this hypothesis. I describe women's knowledge of health, sickness and medicine in an un-acculturated Mexican migrant community in Athens, Georgia, United States, so we might better understand what people of the Mexican diaspora stand to lose with assimilation into mainstream American culture. I also discuss how the dismissal of such cultural knowledge by medical professionals and social scientists can further undermine health in diaspora populations and conclude by proposing the next step in testing this hypothesis. 


\section{RESEARCH SITE}

Athens, Georgia is located 70 miles northeast of Atlanta and is home to approximately 90,000 residents (Adams et al., 1997). Much of Athens's economy depends on students who attend the University of Georgia and several small area colleges. The service industry is the largest employment sector in Athens providing over 40 per cent of its jobs. Other industries in Athens include poultry processing, construction, printing, stone-casting and manufacturing of wood, plastic and rubber products, fabricated metal and machinery. Catholic Social Services has estimated that the Hispanic population of Athens is in excess of 10,000. Like the rest of Georgia, most of the Hispanic population of Athens are migrants from Mexico (Wallace, 2001). The majority of migrants from Latin America reside in close-knit barrios that are relatively isolated and on the outskirts of town (Adams et al., 1997).

My research took place in one of these neighborhoods, which is known by its Spanish-speaking residents as "Los Duplex." Nearly three quarters of the 131 residences in Los Duplex are occupied by Mexican tenants and over half of these households include women and children. Los Duplex stands out from other Mexican neighborhoods in Athens because it has a community center (known locally as "La Escuelita" or little school) that offers English classes, nutrition and parenting discussion groups, and after school activities and homework help for neighborhood children. It also sponsors semi-annual events to celebrate Mexican culture, which has helped cultivate a sense of community among the residents, who come from many different parts of Mexico and have had a variety of life experiences.

However, Los Duplex is a low-income neighborhood. Most men and women in the community are employed by poultry processing plants and other types of factories where they work long hours in difficult environments for relatively low wages (US\$ 7.00-US\$ 9.00 per hour). The rowdy, weekend behavior of single men has led to tension in the community between families and all male households, as well as occasional violence. Gunfire is commonly heard at night in Los Duplex during the weekends. While it is usually just drunks making noise with their firearms, stray bullets are dangerous, especially in a neighborhood where lots of children play outside. A few months after I finished my research there was a fatal shooting in front of La Escuelita one Saturday afternoon. 


\section{RESEARCH PARTICIPANTS AND METHODS}

Women were the focus of my study because they are responsible for the health of their families in both Mexico (Logan, 1983) and Mexican communities in the United States (Bruhn, 1997). Thus, the selection of research participants was restricted to the 51 Los Duplex households that include adult women. I began my fieldwork by volunteering at La Escuelita. This provided an opportunity to collaborate with the director, who has worked with the Mexican community for over five years and to meet a number of families in the neighborhood. Through my role as a volunteer I was able to recruit women from 12 households to participate in the study. I recruited women from an additional 12 households by asking research participants to introduce me to friends and family who might be interested in the research and invited women from another four to participate, who I met on my own. Together these 28 households are made up of 47 men, 52 women, 31 boys and 26 girls. At least one adult woman from the 28 households participated in one or more of the formal data collection activities that I carried out.

None of the 37 women who participated in the research spoke more than a few phrases of English. The majority of my research participants $(92 \%)$ are from the central Mexican states of Michoacán, Guerrero, the Distrito Federal, Hidalgo, Estado de México, Puebla, Guanajuato and Morelos, while the rest are from Nyarit and Tamaulipas. At the time data collection took place, research participants had been living in the United States from six months to 13 years, with an average of 3.9 years. Eighteen women came from rural communities while the other half were from urban areas. Only five women had extended their formal education beyond secondary school and 10 had completed only primary school. All but two research participants were mothers and eight were also grandmothers.

Household income in Los Duplex depends on the number of working adults in a given home. Eleven research participants lived in households with only one or two working adults, 14 lived in households with three or four people working and three lived in households with five or six employed members. The cost of living in Georgia is relatively low compared to the rest of the United States. Nevertheless, many families in Los Duplex periodically have trouble making ends meet, especially if they have children and/or one or more household members are between jobs. Extended families are a great source of help during these times but women from five households included in the research found it necessary 
to seek out charity donations or public assistance during the study period.

All of the data for this study were collected in Los Duplex between April 2002 and June 2003. During my 13 months of fieldwork I conducted a neighborhood census, participant observation, and structured, freelist, pile sort, unstructured and semi-structured interviews. Details of the specific methods used to gather the data presented in this paper are provided below. This research was reviewed and approved by the Institutional Review Board of the University of Georgia. After I thoroughly explained the aims, objectives, methods and benefits of the project each participant signed a consent form, written in Spanish. Likewise, all of my work in Los Duplex, with the exception of conversations with the director of La Escuelita, was conducted entirely in Spanish.

Thirty-seven women participated in structured interviews during which they were asked to evaluate their own health status and that of other members of their households. Although they may not always match the results of detailed clinical evaluations, self-reported assessments of health are good predictors of mortality, specific disease status and disability (Angel et al., 2001). There is also a positive relationship between parental perception of children's health status and children's actual health status (Bruhn, 1997). Research participants were asked to assess their overall health on a scale of one (horrible) to seven (excellent) and to rate how often they get sick on a scale of zero (never) to four (almost always). They were then asked to rate how often their children, husbands and other household members get sick using the same five point scale. Assessment of health status was followed by questions concerning chronic and acute health conditions, the use of different types of medicines and encounters with the mainstream medical system of Athens. I also accompanied seven research participants to 23 medical appointments at the public hospital, the local free clinic, the Public Health Department clinic, a private obstetrics and gynecology specialist, and a private pediatrician to gather further information on migrant experiences with professional medical care.

Thirteen women participated in semi-structured interviews on health and sickness. In these interviews, women were asked to describe healthy and sick people, discuss why people get sick and reflect on what people can do to avoid sickness and maintain health. Nine of the women who were involved in the health interviews also participated in additional semi-structured interviews on medicine (the other four declined to do a 
second interview because of work and family obligations). These interviews included general questions about knowledge, perception and use of both herbal remedies and pharmaceuticals. Semi-structured interviews were tape-recorded upon consent of research participants and their average length was one hour.

Twenty-five women completed freelist interviews, in which they were asked to name all of the herbal medicines they know. While I attempted to collect freelist data on all the non-herbal medicines used in Los Duplex this proved difficult, as most of my research participants were not familiar with the names of prescription drugs. Thus, I compiled my own list of such medicines through daily participant observation in the community. Participant observation was also used to gather data on social relations and networks, as well as to assess the level of social support research participants receive from their neighbors.

\section{HEALTH STATUS}

The women in my study who were under the age of 40 consider themselves to be in good overall health. On the seven-point scale most rated their health a five (Table 1) and only two reported suffering from any chronic condition (gastritis and a faulty heart valve). Most of the women in this age bracket rated the frequency they get sick a "one" or a "two" (Table 2). The most common health problems that these women encounter in Athens are gripas (colds) and indigestion. The 12 research participants who were 40 and older rated their health slightly lower than the younger women did (Table 1). Older women also appear to get sick more often than the younger cohort (Table 2). In women 40 and over, eight reported that they suffer a chronic illness. The illnesses

TABLE 1

SELF-ASSESSMENT OF HEALTH ON A SCALE OF ONE TO SEVEN

\begin{tabular}{|l|cc|}
\hline Rating & Women under 40 & Women 40 and Over \\
\hline 1 (horrible) & - & - \\
2 (very poor) & - & - \\
3 (poor) & - & 2 \\
4 (ok) & 3 & 5 \\
5 (good) & 20 & 5 \\
6 (very good) & 1 & - \\
7 (excellent) & 1 & - \\
\hline
\end{tabular}


TABLE 2

THE FREQUENCY MEXICAN MIGRANT WOMEN GET SICK

\begin{tabular}{|l|cc|}
\hline Rating & Women under 40 & Women 40 and Over \\
\hline 0 (never) & 1 & - \\
1 (almost never) & 11 & 4 \\
2 (sometimes) & 12 & 6 \\
3 (often) & 1 & 2 \\
4 (almost always) & - & - \\
\hline
\end{tabular}

TABLE 3

THE FREQUENCY CHILDREN AND ADULT HOUSEHOLD MEMBERS GET SICK, ACCORDING TO MEXICAN MIGRANT WOMEN

\begin{tabular}{|l|rc|}
\hline Rating & Adults & Children \\
\hline 0 (never) & 1 & 2 \\
1 (almost never) & 11 & 7 \\
2 (sometimes) & 22 & 16 \\
3 (often) & 3 & 5 \\
4 (almost always) & - & - \\
\hline
\end{tabular}

reported were diabetes, heart disease, hypertension, asthma and migraine.

The health of other adult household members was generally perceived to be good (Table 3). The most commonly reported health problems of adult household members were gripas and allergies. However, three research participants have husbands with diabetes, two have husbands with asthma symptoms, two have husbands with high cholesterol and another woman's husband suffered a heart attack during the study period. The 30 research participants who live in Athens with children under the age of 18 perceived them to be healthy overall. Mexican children were reported to suffer common childhood ailments like gripas, ear infections, fevers and rashes. However, four of my research participants had children with asthma symptoms that became quite serious at times and another two reported that their sons had kidney problems.

\section{MAINTAINING AND PROMOTING HEALTH}

All 13 women who participated in the health interview appreciate the fact that health must be maintained. Eight talked about the importance 
of good dietary practices, because a good diet can help you avoid getting sick. Guendelman and Abrams (1995) found that women who were born in Mexico consume more protein, vitamins A and C, folic acid and calcium than both Mexican women born in the United States and Americans of European descent. This is reflected in my research participants' definition of a good diet, which includes vegetables, fruits and plenty of water. Research participants defined a poor diet as one that is high in fats, sodas, sweets, chips and meat, as well as foods that are not fresh.

The nutritional composition of the diet alone is not enough to ensure the benefits of healthy eating. One must eat foods that agree with the body and should be mindful of balancing the humoral ${ }^{1}$ qualities of food and drink with the body's humoral condition. Two women also pointed out that it is important not to overeat. Eating outside the home, especially "in the street" when one does not have the time or the money to cook a proper meal is thought to lead to poor dietary habits and subsequent sickness. All of the women who participated in the study reported that they cook traditional Mexican foods for their families. I ate at least one meal in 18 out of 28 households involved in the research. The food that is prepared by women in Los Duplex typically consists of tortillas, beans, and salads, as well as soups and stews made with fresh chiles, tomatoes, onions and meat.

In addition to a good diet, the women I interviewed mentioned several other behaviors that contribute to the maintenance of good health, including exercising and staying active, being in a good mood, caring for oneself and others and paying attention to the body. Five women mentioned exercise specifically and another explained that if you are active in your job and daily routine, there is no need to do additional exercises. Indeed, using NHANES III data King et al. (2001) found that having a physically active occupation, such as waiting tables, cleaning and working in agriculture, factories or construction decreases one's likelihood of being obese. For people who engage in little to no leisure time physical activity, the chances of being obese can be cut in half with a physically active occupation. ${ }^{2}$

According to Mexican women, staying calm and avoiding anger contribute to good health as does thinking happy thoughts. Paying attention to the body helps one to notice signs of sickness before they become serious and allows one to eat foods and do exercises that are in agreement with the body. Getting enough sleep is also considered an essential part of taking care of oneself. Three women stressed that it is important to 
enjoy the company of and care for friends and family. Advice for maintaining health also included engaging the mainstream healthcare system. Five women emphasized the importance of getting regular medical check-ups, because sometimes we might be sick without realizing it.

Eating a well-balanced diet, staying active, avoiding stress and emotional upset and paying attention to the body are all things that any competent medical professional would advise. Yet despite their knowledge of health, Mexican women also explained how difficult it is to avoid all of the things that can make you sick in the United States. The climate of Georgia was a particular concern and was discussed by nearly all the Mexican migrants who I talked to during my fieldwork. Extremes of heat and cold, as well as fluctuations between the two, which typify the weather of Athens, are believed to cause sickness by people of Mexican descent (Clark, 1959; Kay, 1977). Several women expressed a longing for the more agreeable climate of central Mexico and some who had lived in California before moving to Athens explained that although there were more jobs in Georgia, they preferred California because the climate reminded them of home. Likewise, most of the women who I interviewed about health expressed their dissatisfaction with food quality and availability in the United States. As one woman explained, while fresh fruits and vegetables are among the least expensive foods in Mexico, in the United States produce is expensive and it's difficult not to be temped by all the "great tasting" but unhealthy foods that are cheap and readily available.

\section{MEDICINE AND MEDICAL CARE}

Recommending and/or administering medicine to sick family members is a responsibility and a skill that the women of all 28 households included in my study share. The majority of medicines used by my research participants are home remedies, which include both medicinal herbs and over the counter medications. However, with rare exception, prescription drugs are also used from time to time in Mexican households. Although such drugs are selected by a medical doctor, Mexican families expect women to learn how much of and how often a prescription drug should be taken and to make sure sick family members take their medicine.

A total of 42 plants were included in the 24 freelists and 16 of these were mentioned by at least two women. Most of these species were 
introduced to Mexico by the Spanish in the 16th century. The two most frequently mentioned herbs, manzanilla (chamomile) and yerbabuena (mint) are prepared as infusions or teas for stomach problems by women in Los Duplex and other Mexican American communities (Curtin, 1965; Kay, 1977). These two plants were also included among the most common herbal remedies cited in both Clark (1959) and Rivera et al. (2002). Ruda (rue) and albahaca (basil) were the third and fourth most frequently mentioned herbs in the freelist data. Clark (1959) also listed these plants among the most common medicinal herbs in the community she studied and they are both included in Curtin's (1965) ethno-botanical study of New Mexico. In Los Duplex they are usually soaked in rubbing alcohol to make a topically applied liniment for aches and pains. The efficacy of the most popular herbal medicines that women bring from Mexico to the United States is well documented in the chemical and pharmacological literature (Waldstein, 2006). For example, chamomile (Matricaria chamomila) has demonstrated a variety of properties in laboratory tests (including antibacterial, antispasmodic and anti-inflammatory activity) useful for the treatment of gastro-intestinal maladies (Ross, 2001). Nevertheless, four women who I interviewed about medicines questioned the efficacy of medicinal plants available in the United States. In Mexico, fresh plant materials are widely available and desirable because they are perceived to be more potent than dried plants. While a few commonly used species are easy to grow in Athens, Mexican women lamented the fact that only dried herbs are available at the local Mexican grocery.

Herbal medicines are an essential component of the Los Duplex pharmacopoeia, but they are not the only home remedies used. In Mexico there is a strong cultural tradition of purchasing patent medicines at pharmacies (Logan, 1983). Use of the two most popular non-prescription commercial medicines, Vick's Vaporub and Tylenol (paracetemol) was also documented in Kay's (1977) study. Vaporub is used for congestion in the chest and sinuses and Tylenol is popular for headaches and fevers in some families. One woman who I interviewed said that she preferred Advil (ibuprofen) to Tylenol, but aspirin was not very popular. Pomadas (salves) from Mexico are used for burns, cuts and pain. Some of my research participants also use Alka Seltzer (or simply baking soda) for certain stomach symptoms. These commercially produced home remedies are purchased at Mexican and American groceries and pharmacies.

The most common medicines prescribed to people in Los Duplex are cough syrups, anti-fungal creams, antibiotics, anti-hypertensives and 
diabetes medications. In Los Duplex, children covered by Medicaid ${ }^{3}$ are given more prescription medicines than adults because they go to the doctor more frequently. However, uninsured adults and children can and do get prescriptions from the free clinic, public hospital and public health department when needed. Prescriptions are usually filled at the pharmacy closest to Los Duplex, which accepts Medicaid. However, patients of the free clinic can get their prescriptions filled for free only at one specific pharmacy located near the public hospital.

Although a whole range of medicines, from medicinal plants to prescription drugs, are available to Mexican families in Los Duplex, structured and semi-structured interview data reveal a preference for herbal remedies. Fourteen of the women who participated in the structured interviews indicated that they use herbal and other home remedies more often than medicines recommended and/or prescribed by a physician. Another 11 stated that they use home remedies and pharmaceutical medicines with equal frequency and only 12 indicated a preference for doctor recommended drugs. Semi-structured data provide further evidence of a preference for home remedies, at least for mild disorders that are not considered life threatening. Herbal medicines are considered safe for the body because they are natural, while pastillas (pills) have quimicos (chemicals) in them that can cause side effects. Pastillas are also considered potentially dangerous because they're similar to drogas (illegal drugs) that are addictive and mask serious symptoms. This perception of pharmaceuticals makes them unattractive to many women in Los Duplex and some even claim to never use them. In contrast, all of the women who worked with me had some experience with medicinal herbs and agreed that they are effective, natural and free from side effects.

Despite this preference for herbal medicines, all of the women who I interviewed recognized that sometimes self-medication alone is not effective and professional medical treatment is necessary. While going to the doctor is generally reserved for serious ailments that do not respond to home remedies, my research participants view Athens as a city with extensive professional healthcare resources. At least two women's clinics, two free clinics, two doctor's offices and both hospitals have interpreters on staff and the public hospital has a well-funded low-income assistance program that does not require applicants to be in the country legally. This program covers all hospital expenses for eligible applicants for one year and can be reapplied for on a yearly basis as needed. I have never met anyone from Los Duplex who applied for the program and was turned down. The hospital also employs social workers (some bilingual) 
TABLE 4

CONDITIONS FOR WHICH WOMEN IN LOS DUPLEX REPORTED SEEKING PROFESSIONAL MEDICAL CARE

\begin{tabular}{|l|c|}
\hline Condition & Frequency \\
\hline Birth & 15 \\
Pre-natal Care & 15 \\
General Check-up & 4 \\
Diabetes & 3 \\
Flu & 2 \\
Gastritis & 2 \\
Hypertension & 2 \\
Pregnancy Complication & 2 \\
Chronic Cough & 1 \\
Dizziness & 1 \\
Heart Condition & 1 \\
High Cholesterol & 1 \\
\hline
\end{tabular}

who help new mothers enroll their children in Medicaid, Peachcare ${ }^{4}$ and the Women, Infants and Children (WIC) ${ }^{5}$ program and put single moms in touch with other social workers. Three of the 37 women who did a structured interview have been to a private doctor in Athens. Thirty have been to one of the public hospital or local free clinics at least once. Moreover, twenty women have been admitted to the hospital. Fifteen of the women who I interviewed have given birth to at least one child in Athens and all have taken their young children to a Spanish-speaking pediatrician's office for vaccinations. Five have taken their young children to this doctor for additional concerns. The conditions for which research participants reported seeking professional medical treatment are summarized in Table 4.

\section{SOCIAL NETWORKS AND SOCIAL SUPPORT}

As described above, the women who I interviewed identified spending time with friends and family as important for maintaining health. This bit of folk medical knowledge is also supported by the scientific literature. Informal social networks, social activities and participation in organizations are all associated with better health chances (Berkman and Syme, 1979; Cattell, 2001). One reason why social networks have a protective effect on health is the fact that they're a source of social support, which is influential in maintaining health and preventing sickness by helping people cope with medical problems. Various forms of social 
support (e.g., emotional support, advice, guidance, material aid) have a stress-buffering effect that can improve health and social functioning. Social support is also related to longevity in the epidemiological literature (Hurdle, 2001).

Most Mexican migrants in the United States are part of well-developed social networks that provide them with a great deal of social support. For example, a survey of 679 Mexican immigrant women in San Diego, California found that most respondents were not isolated from their families as a result of migration and that about two-thirds developed friendships after migrating to California. Early access to family support among immigrant women was key to successful adaptation to life in the United States (Vega et al., 1991). Mexican women in Los Duplex are also part of elaborate social networks. Most of the women I worked with have family who they see on a daily basis and I observed near constant mutual support (from a sympathetic ear to financial assistance) among members of extended families. I also watched relationships develop between neighbors who come from completely different parts of Mexico. Sometimes children set this process in motion. Mothers become friendly with each other if their children regularly play together on the playground, at "La Escuelita" or at school. Vecinos (people who inhabit the two apartments of a duplex) also become quite friendly with each other, as do people who live a few doors down or across the street from one another. Social support among women in Los Duplex includes favors, such as providing lifts to the store, hiring each other to cater parties and taking care of each other's children.

Migrant social networks are also important sources of information. Most anthropological and sociological research on Mexican migration to the United States indicates that migrant social networks have made this large-scale migration flow possible. Ties to current or former migrants are used to reduce the costs of migration and increase the likelihood of finding a good job in the United States (Massey and Espinosa, 1997; Wilson, 2000). Information about healthcare services in host communities also flows through Mexican women's social networks (ChaviraPrado, 1992).

Potential barriers to professional healthcare services cited by women in Los Duplex include the high cost of medical care and a lack of health insurance, poor English language skills and difficulty finding transportation. Thus, accessing professional medical care in Athens generally involves learning about the few social services for which undocumented 
migrants are eligible, as well as healthcare providers that accept and/or offer such services. Women must then find out where these institutions are located, arrange rides to pick up and drop off applications and find someone who speaks English to help fill out applications and interpret during appointments. While husbands sometimes help their wives by asking co-workers about various services, driving women to appointments, or arranging other means of transportation, women rely more on their own networks of female family and friends. The women who I interviewed identified friendships as a source of assistance when they determine that they, or members of their families, need professional medical care. Eight of the women explained that friends and family who are established in Athens help newcomers find inexpensive and/or bilingual healthcare services when needed.

\section{DIASPORA, HEALTH AND DIFFERENT MEDICAL TRADITIONS}

The people of Los Duplex are part of a Mexican diaspora as demonstrated by the bounded nature of their community and social networks, as well as their faith in Mexican health beliefs and practices. Although the women who I worked with befriend people from all over Mexico, they have limited contact with the English-speaking majority of Athens. Likewise, their social networks include few, if any Spanish-speaking people from other parts of Latin America. People from Los Duplex patronize the Mexican owned businesses of Athens and work in occupations where they are surrounded by their compatriots. Furthermore, the majority of the women who I interviewed expressed a desire to eventually return to Mexico. Part of this longing for home revolves around the perception that it is easier to follow principles of good health in Mexico where both fresh produce and medicinal plants are readily available and the climate is more agreeable. Yet despite the difficulties of staying healthy in United States, the diaspora actually has a positive effect on health as cultural distinction from the rest of American society ensures the persistence of traditional Mexican medical knowledge. Likewise, the Mexican social networks that tie migrants to each other and to their homeland reduce the health risks that are generally associated with migration.

Of course, migrants and their descendents are far from homogenous and the medical knowledge held by women in Los Duplex may not necessarily be shared by all Mexicans in the United States. For example, there is preliminary research that suggests Mexican men who tra- 
vel to the United States by themselves suffer from malnutrition because they don't have wives with them who make sure they eat well (Orozco, 2003). Much discussion in the social science, public health, nursing and medical literature has been devoted to minimizing such health risks associated with migration and diaspora. Most often this involves removing barriers to biomedical services and otherwise integrating diasporas into host societies. As well intentioned as these efforts may be, they are limited by the implicit assumptions that biomedicine is a prerequisite to human health and that host societies are healthier than their migrant populations.

In fact, Mexican migrants and their descendants in the United States are healthier than middle-class American citizens of all other ethnicities (Iannota, 2003; Morales et al., 2002; Vega and Amaro, 1994) and this is, at least in part, due to their traditional medical knowledge. Members of the Mexican diaspora and recent migrants in particular, underutilize professional health services when compared to other segments of the American population (Vega and Amaro, 1994). This has been attributed to linguistic, financial, structural and cultural barriers to the mainstream American medical system (Chavez, 1986; Iannota, 2003; Morales et al., 2002; Saunders, 1954; Warda, 2000). However, the Los Duplex case suggests that Mexican women may actually learn how to access professional healthcare services via their social networks, but in many cases may opt not to seek care from doctors because they are not interested in the types of medicines they offer, especially if appropriate herbal remedies are available. Going to the doctor is generally a last resort, used only when self-treatment proves inadequate. Thus, rather than improving the health of Mexicans by assimilating them into the mainstream American medical system, a better use of resources would be funding public health campaigns that encourage Mexican women to pass on their knowledge of health, sickness and medicine to subsequent generations.

Educating American medical professionals about Mexican health beliefs and practices is also important, because it will allow them to better serve those migrants who do seek their advice. Based on the 23 medical appointments that I attended with women from Los Duplex, it seems that healthcare professionals in Athens are not aware that most Mexicans routinely use herbal remedies. None of the healthcare providers that I observed asked their Mexican patients about the use of herbal medicines, though a few did ask about the use of non-prescription drugs. This is potentially dangerous because even when they are pre- 
scribed medications Mexican women may continue to take the home remedies they tried as a first resort, which can lead to adverse drug interactions (Rivera et al., 2002).

Social scientists interested in migration and health have also given little attention to a growing trend of reduced dependence on professional medical services by the acquisition of health knowledge and self-care skills for health maintenance (Giachello, 1994). I have found that Mexican migrant women know enough about health, sickness and medicine to successfully manage the health of their families without professional assistance (at least most of the time). The ethnomedical and ethnopharmacological literature indicates that Mexicans are not unique in their understanding of health, sickness and medicine. People all over the world have traditional medical beliefs and practices that keep them healthy, even in places where biomedical resources are scarce. Interdisciplinary projects that link diaspora studies with ethnomedicine could play a critical role in improving the health of migrants and their descendents by preserving and/or reviving these traditions when populations disperse and move. This would also be a much needed complement to efforts aimed at increasing access to professional medical services.

\section{CONCLUSIONS}

In sum, Los Duplex is a poor neighborhood, yet most of the people who live there with their families are in good physical and emotional condition. Although older women perceive themselves to be in slightly worse health than do younger women, self-assessments and assessments of other household members were generally positive. Few of the women in this study suffer from chronic and/or life-threatening health problems. Spouses and children are also described as healthy. Women encourage healthy behaviors such as eating nourishing foods that agree with the body, exercising and staying active, maintaining a positive attitude and spending time with friends and family. All of these things contribute to good health because they reduce stress and prevent depression. When members of their families do get sick, the Mexican women of Los Duplex recommend and administer home remedies and oversee the use of prescription drugs. The efficacy of the most popular herbal medicines is well supported by the botanical, chemical and pharmacological literature for the kinds of conditions they treat in Los Duplex. 
Most of the women who I interviewed have family who they see daily and all have several friends and acquaintances. The social networks that Mexican women maintain and expand are a valuable source of social support. Women are of great importance in keeping members of Mexican families close. They thereby ensure that relatives may still be relied upon for emotional and financial assistance during serious illness, despite acculturative pressures. In addition, information about hospitals, clinics, doctors, interpreters, social workers, food banks, church organizations and government officials travels through these social networks. Thus, the migration networks that draw Mexican women to Los Duplex also help migrant families access the mainstream American health care system and the little public assistance for which they are eligible.

This case study shows that diaspora need not always lead to disease. Though migration is fraught with health risks and marginalization from mainstream society can reduce access to healthcare services, many of the world's cultural traditions include knowledge of health, sickness and medicine that is effective in preserving human health. I have documented several aspects of Mexican migrant culture that provide some protection against poverty and low educational status as well as medical beliefs and practices that reduce dependence on professional health care services. The important role that traditional medical knowledge plays in the overall health management practices of migrants in Los Duplex suggests that the loss of such knowledge could lead to a decline in health. Future research is needed to determine how well traditional medical knowledge is preserved in more acculturated Mexican communities and whether an abandonment of traditional medical practices is indeed associated with poorer health. In the meantime, it seems safe to say that promoting cultural beliefs and practices that have a positive impact on health is as important, if not more so, as integrating diaspora populations into their host societies.

\section{ACKNOWLEDGEMENTS}

This research was funded by a National Science Foundation Doctoral Dissertation Improvement Award (BCS-0090216) and conducted under the guidance of Elois Ann Berlin. Many thanks to all of the people in Los Duplex who were willing to work with me and to Paru Raman, Ian Harper, Roy Ellen and two anonymous reviewers for reading and commenting on earlier drafts of this paper. 


\section{NOTES}

1. Humoral theory is based on the work of Hippocrates, Galen and Avicena and continues to flourish in many parts of the world, especially Asia and Latin America. Currently, in most humoral systems found today, health is a state of balance between "hot" and "cold" humors. Sickness results from imbalance and treatments (foods or medicines) are designed to restore balance.

2. The study by King et al. is exceptional in that it considers incidental, transportational and occupational activities. As a population, Hispanics have been characterized in the public health literature as sedentary, because they engage in less leisure time physical activity than other Americans. Failure to recognize cultural and class differences in activity patterns can lead to patient profiling and limit the ability of healthcare professional to effectively serve Hispanics.

3. Medicaid is a jointly funded Federal and State government program that pays for medical assistance for certain individuals and families with low incomes and resources. Currently only United States citizens are eligible for Medicaid.

4. Peachcare is the state of Georgia's health insurance program for lowincome children born in the United States. Many Mexican families prefer Peachcare over Medicaid because Peachcare applications need to be renewed less often.

5. The Special Supplemental Nutrition Program for Women, Infants, and Children, better known as the WIC Program, is administered by the Food and Nutrition Service and provides nutritious foods to supplement diets, information on healthy eating and referrals to healthcare services for lowincome women, infants and children up to age five who are at nutritional risk. This program is not exclusive to American citizens.

\section{REFERENCES}

Amaro, H., et al.

1990 "Acculturation and marijuana and cocaine use: findings from HHANES 1982-84", American Journal of Public Health, 80(Suppl.): 54-60.

Angel, J.L., et al.

2001 "Nativity and self-assessed health among pre-retirement age Hispanics and Non-Hispanic Whites", International Migration Review, 35(3): 784-803.

Berkman, L.F., and S.L. Syme

1979 "Social networks, host resistance, and mortality: a nine-year follow-up study of Alameda County residents", American Journal of Epidemiology, 109(2): 186-204. 
Bronfman, M.

1998 "Mexico and Central America", International Migration, 36(4): 609-642.

Brubaker, R.

2005 "The 'diaspora' diaspora", Ethnic and Racial Studies, 28(1): 1-19.

Bruhn, J.G.

1997 "Health: its meaning and expression", in J.G. Bruhn and J.E. Brandon (Eds), Border Health: Challenges for the United States and Mexico, Garland Publishing, Inc., New York.

Carballo, M., and A. Nerukar

2001 "Migration, refugees, and health risks", Emerging Infectious Diseases, 7(3): 556.

Cattell, V.

2001 "Poor people, poor places, and poor health: the mediating role of social networks and social capital", Social Science and Medicine, 52: $1501-1516$.

Chavez, L.R.

1986 "Mexican immigration and health care: a political economy perspective", Human Organization, 45(4): 344-352.

Chavira-Prado, A.

1992 "Work, health, and the family: gender structure and women's status in an undocumented migrant population", Human Organization, 51(1): 53-64.

Clark, M.

1959 Health in the Mexican-American Culture, University of California Press, Berkeley.

Curtin, L.S.M.

1965 Ethnobotany of Spanish Speaking New Mexico, Southwest Museum, Los Angeles.

Friedman-Jimenez, G., and J.S. Ortiz

1994 "Occupational health", in C. Molina and M.A. Molina (Eds), Latino Health in the US: A Growing Challenge, American Public Health Association, Washington, DC.

Giachello, A.L.M.

1994 "Issues of access and use", in C. Molina and M.A. Molina (Eds), Latino Health in the US: A Growing Challenge, American Public Health Association, Washington, DC.

Gonzalez Gutierrez, C.

1999 "Fostering identities: Mexico's relation with its diaspora", The Journal of American History, 86(2): 545-567.

Guendelman, S., and B. Abrams

1995 "Dietary intake among Mexican-American women: generational differences and a comparison with White Non-Hispanic women", American Journal of Public Health, 85: 20-25. 
Hurdle, D.E.

2001 "Social support: a critical factor in women's health and health Kay, M.A. promotion", Health and Social Work, 26(2): 72-79.

1977 "Health and illness in a Mexican American barrio", in E.H. Spicer (Ed.), Ethnic Medicine in the Southwest, The University of Arizona Press, Tucson.

King, G.A., et al.

2001 "Relationship of leisure-time physical activity and occupational activity to the prevalence of obesity", International Journal of Obesity, 25: 606-612.

Logan, K.

1983 "The role of pharmacists and over the counter medications in the health care system of a Mexican City", Medical Anthropology, summer: 68-84.

Marin, G., et al.

1989 "Cigarette smoking among San Francisco Hispanics: the role of acculturation and gender", American Journal of Public Health, 79: 196-198.

Massey, D.S., and K.E. Espinosa

1997 "What's driving Mexico-U.S. migration? a theoretical, empirical, and policy analysis", American Journal of Sociology, 102(4): 939999.

Molina, C., et al.

1994 "The influence of culture, class, and environment on health care", in C. Molina and M.A. Molina (Eds), Latino Health in the US: A Growing Challenge, American Public Health Association, Washington, DC.

Morales, L.S., et al.

2002 "Socioeconomic, cultural, and behavioral factors affecting Hispanic health outcomes", Journal of Health Care for the Poor and Underserved, 13(4): 477-503.

Orozco, R.

2003 "Mexican testimonies of illness and healing: a generational analysis", paper Presented at the 63rd Annual Meeting of the Society for Applied Anthropology, Portland, Oregon, March 19-23.

Pieroni, A., et al.

2005 "Traditional phytotherapy and trans-cultural pharmacy among Turkish migrants living in Cologne, Germany", Journal of Ethnopharmacology, 102: 69-88.

Rivera, J.O., et al.

2002 "Evaluation of the use of complementary and alternative medicine in the largest United States-Mexico border city", Pharmacotherapy, 22(2): 256-264. 
Ross, I.

2001 Medicinal Plants of the World: Chemical Constituents, Traditional and Modern Medicinal Uses, Humana Press, Totowa, NJ.

Saunders, L.

1954 Cultural Differences in Medical Care: The Case of the SpanishSpeaking People of the Southwest, Russell Sage Foundation, New York.

Scribner, R., and J.H. Dwyer

1989 "Acculturation and low birth weight among Latinos in the Hispanic HANES", American Journal of Public Health, 79: 12631267.

Sundquist, J., and M.A. Winkleby

1999 "Cardiovascular risk factors in Mexican American adults: a transcultural analysis of NHANES III, 1988-1994", American Journal of Public Health, 89(5): 723-730.

Tseng, M., et al.

2000 "Country of birth and prevalence of gallbladder disease in Mexican Americans", Ethnicity \& Disease, 10(1): 96-105.

Vega, W.A., and H. Amaro

1994 "Latino outlook: good health, uncertain prognosis", Annual Review of Public Health, 15: 39-67.

Vega, W.A., et al.

1991 "Social networks, social support, and their relationship to depression among immigrant Mexican women", Human Organization, 50: $154-162$.

Waldstein, A.

2006 "Mexican migrant ethnopharmacology: pharmacopoeia, classification of medicines and explanations of efficacy", Journal of Ethnopharmacology, 108: 299-310.

Waldstein, A., and C. Adams

2006 "The interface between medical anthropology and medical ethnobiology", Journal of the Royal Anthropological Institute, 12: 95118.

Wallace, J.K.

2001 Cultural Conceptualizations of HIV and AIDS Among Female Mexican Immigrants, Master of Arts Thesis, University of Georgia, Athens.

Warda, M.

2000 "Mexican Americans' perceptions of culturally competent care", Western Journal of Nursing Research, 22(2): 203-225.

Wilson, T.D.

2000 "Anti-immigrant sentiment and the problem of reproduction/maintenance in Mexican immigration to the United States", Critique of Anthropology, 20(2): 191-213. 